\title{
RESEARCH
}

Open Access

\section{TFE3 fusions escape from controlling of mTOR signaling pathway and accumulate in the nucleus promoting genes expression in Xp11.2 translocation renal cell carcinomas}

Xiaoqin Yin ${ }^{1,2}$, Bo Wang ${ }^{1,2}$, Weidong Gan ${ }^{3 *}$, Wenyuan Zhuang ${ }^{3}$, Zou Xiang ${ }^{4}$, Xiaodong Han ${ }^{1,2^{*}}$ and Dongmei $\mathrm{Li}^{1,2^{*}}$

\begin{abstract}
Background: Xp11.2 translocation renal cell carcinoma (tRCC) is mainly caused by translocation of the TFE3 gene located on chromosome Xp11.2 and is characterized by overexpression of the TFE3 fusion gene. Patients are diagnosed with tRCC usually before 45 years of age with poor prognosis. We investigated this disease using two tRCC cell lines, UOK109 and UOK120, in this study.

Methods: The purpose of this study was to investigate the pathogenic mechanism of TFE3 fusions in tRCC based on its subcellular localization, nuclear translocation and transcriptional activity. The expression of TFE3 fusions and other related genes were analyzed by quantitative reverse transcription PCR (qRT-PCR) and Western blot. The subcellular localization of TFE3 was determined using immunofluorescence. The transcriptional activity of TFE3 fusions was measured using a luciferase reporter assay and ChIP analysis. In some experiments, TFE3 fusions were depleted by RNAi or gene knockdown. The TFE3 fusion segments were cloned into a plasmid expression system for expression in cells.

Results: Our results demonstrated that TFE3 fusions were overexpressed in tRCC with a strong nuclear retention irrespective of treatment with an mTORC1 inhibitor or not. TFE3 fusions lost its co-localization with lysosomal proteins and decreased its interaction with the chaperone 14-3-3 proteins in UOK109 and UOK120 cells. However, the fusion segments of TFE3 could not translocate to the nucleus and inhibition of Gsk3 $\beta$ could increase the cytoplasmic retention of TFE3 fusions. Both the luciferase reporter assay and ChIP analysis demonstrated that TFE3 fusions could bind to the promoters of the target genes as a wild-type TFE3 protein. Knockdown of TFE3 results in decreased expression of those genes responsible for lysosomal biogenesis and other target genes. The ChIP-seq data further verified that, in addition to lysosomal genes, TFE3 fusions could regulate genes involved in cellular responses to hypoxic stress and transcription.
\end{abstract}

(Continued on next page)

\footnotetext{
* Correspondence: gwd@nju.edu.cn; hanxd@nju.edu.cn; lidm@nju.edu.cn ${ }^{3}$ Department of Urology, Affiliated Drum Tower Hospital of Medical School of Nanjing University, Nanjing 210008, Jiangsu, China

${ }^{1}$ Immunology and Reproduction Biology Laboratory \& State Key Laboratory of Analytical Chemistry for Life Science, Medical School, Nanjing University, Nanjing 210093, Jiangsu, China

Full list of author information is available at the end of the article
}

(c) The Author(s). 2019 Open Access This article is distributed under the terms of the Creative Commons Attribution 4.0 International License (http://creativecommons.org/licenses/by/4.0/), which permits unrestricted use, distribution, and reproduction in any medium, provided you give appropriate credit to the original author(s) and the source, provide a link to the Creative Commons license, and indicate if changes were made. The Creative Commons Public Domain Dedication waiver (http://creativecommons.org/publicdomain/zero/1.0/) applies to the data made available in this article, unless otherwise stated. 
(Continued from previous page)

Conclusions: Our results indicated that the overexpressed TFE3 fusions were capable of escaping from the control by the mTOR signaling pathway and were accumulated in the nucleus in UOK109 and UOK120 cells. The nuclear retention of TFE3 fusions promoted the expression of lysosomal genes and other target genes, facilitating cancer cell resistance against an extreme environment.

Keywords: TFE3, mTOR, Subcellular localization, Nuclear retention, Lysosome, Transcription factor,

\section{Background}

Xp11.2 translocation renal cell carcinoma (Xp11.2 tRCC), also known as TFE3-fusion associated RCC, was listed as a new entity in the 2004 WHO classification of renal tumors and was recently included in the MiT (microphthalmia transcription factor) family tRCC as a new subset of RCC in the 2016 WHO classification [1, 2]. TFE3-fusion associated RCC is defined by several different translocations involving chromosome Xp11.2, including $A S P L$, PRCC, NonO, CLTC, SFPQ1, LUC7L3, KHSRP, PARP14, $D V L 2$, and $R B M 10$, as well as unknown genes on chromosome 10 [3-8]. All these resulted in gene fusions involving the Transcription Factor Binding to IGHM Enhancer 3 (TFE3) gene and Xp11.2 tRCC is characterized by overexpressed TFE3 fusion gene and affecting patients younger than 45 years with poor prognosis.

MiT family of transcription factors which encodes four distinct genes, including MITF, TFEB, TFE3, and TFEC, contains the basic helix-loop-helix (bHLH) structure and is capable of recognizing the transcription initiation or E-box (Ephrussi boxes) sites (CANNTG) in the genome. More recently, MITF, TFEB, and TFE3 have been identified as regulators of lysosomal function and metabolism. They can recognize numerous lysosomal and autophagy genes with one or more 10-base pair motifs (GTCACGTGAC) termed as Coordinated Lysosomal Expression and Regulation (CLEAR) elements, which in turn promotes gene transcription $[9,10]$. To respond to the changes in the levels of nutrients within cells, TFE3 can regulate its intracellular distribution through activation or inactivation in an mammalian target of rapamycin complex 1 (mTORC1)-dependent manner $[10,11]$. In fully fed cells, TFE3 is recruited to the lysosomal surface, where TFE3 undergoes mTORC1-dependent phosphorylation through interaction with active Rag GTPases. Active mTORC1 phosphorylates TFE3 at serine 321 (Ser321) residue to create a binding site for the cytosolic chaperone 14-3-3. Interaction with 143-3 proteins results in sequestration of TFE3 in the cytosol $[10,12,13]$. Conversely, when nutrients are scarce, inactivation of mTORC1, together with Ser321 dephosphorylation, prevents TFE3 proteins from binding to $14-3-3$, resulting in the rapid accumulation of TFE3 in the nucleus. TFE3 thus regulates in the nucleus the expression of targeted genes, such as 4EBP3 which can repress translation initiation. Furthermore, depletion of endogenous TFE3 entirely abolishes the response of cells to starvation and overexpression of TFE3 triggers lysosomal exocytosis [10].

In Xp11.2 tRCC, the encoded fusion TFE3 proteins retains the bHLH structure for DNA binding, suggesting that these overexpressed fusion TFE3 proteins may function as oncogenic transcription factors. Up to now, the most widely accepted model that accounts for TFE3-fusion-mediated oncogenesis argues for the availability of a constitutively active promoter leading to an abnormal TFE3 transcriptional activity $[3,14,15]$. A multiple of molecular pathways well-implicated in carcinogenesis are regulated in part by the TFE3 protein, including activation of TGF- $\beta$ and the ETS transcription factors, E-cadherin expression, CD40L-dependent lymphocyte activation, folliculin signaling, and mTORC1 signaling [16-19]. However, the intracellular distribution and specific functions of TFE3-fusion proteins in Xp11.2 tRCC still remain unknown, and thus, there is no effective treatment for patients with this RCC subtype.

In this study, we focused on two Xp11.2 tRCC cell lines, UOK109 from NonO-TFE3 fusion and UOK120 from PRCC-TFE3 fusion tRCC, to explore intracellular distribution of the fusion TFE3 proteins and possible pathogenic mechanisms as a result of changes in intracellular localization of the fusion TFE3 proteins. We found that highly overexpressed TFE3 fusions showed strong nuclear accumulation in Xp11.2 tRCCs and were capable of escaping from the control by the mTOR signaling pathway. Furthermore, the nuclear retention of TFE3 fusions could regulate the expression of lysosomal biogenesis genes, thus promoting cancer cells resistance against an extreme environment.

\section{Methods \\ Cell culture}

The cell lines used in this study included 786-0 human kidney adenocarcinoma cells (ATCC ${ }^{\bullet}$ CRL-1932 $2^{\mathrm{m}}$ ), HK-2 human kidney cortex/proximal tubule (ATCC $^{\circ}$ CRL2190 ${ }^{\mathrm{rm}}$ ), and the renal carcinoma cell lines UOK120 and UOK109 (gifts of Dr. W. Marston Linehan, National Cancer Institute, Bethesda, MD). The UOK120 and UOK109 cell lines were derived from primary papillary cell carcinoma as described [20], and were derived from 
tumors arising in a 30- and a 39-year-old male, respectively. Cells were cultured in DMEM (Gibco, Grand Island, NY) supplemented with 10\% FBS (Gibco) and 1\% penicillin/streptomycin (Invitrogen, Carlsbad, CA).

\section{Antibodies}

The antibodies used in this study included: mouse anti-GAPDH (Santa Cruz Biotechnology, sc-365,062), goat anti-TFE3 (Santa Cruz Biotechnology, sc-5958), rabbit anti-TFE3 (Abcam, ab93808), mouse anti-4EBP3 (Santa Cruz Biotechnology, sc-134,232), rabbit anti-pS6 (Cell Signaling Technology, 2215), rabbit anti-RPS6 (Abcam, ab40820), rabbit anti-mTOR (Cell Signaling Technology, 2972), rabbit anti-14-3-3 $\beta / \alpha$ (Cell Signaling Technology, 9636), rabbit anti-14-3-3 $\gamma$ (Cell Signaling Technology, 5522), rabbit anti-14-3-3 $\zeta / \delta$ (Cell Signaling Technology, 7413), rabbit anti-14-3-3 $\varepsilon$ (Cell Signaling Technology, 9635), rabbit anti-14-3-3 т (Cell Signaling Technology, 9638), rabbit anti-14-3-3 $\eta$ (Cell Signaling Technology, 5521), rabbit anti-LAMP2 (Cell Signaling Technology, 49,067), rabbit anti- $\beta$-Tubulin (Cell Signaling Technology, 2146), HRP-conjugated goat anti-rabbit (Cell Signaling Technology, 7074), goat anti-mouse secondary antibody (Boster, BA1050), rabbit anti-goat secondary antibody (Boster, BA1060), Alexa Fluor 488-conjugated goat anti-rabbit (Abcam, ab150077), and Alexa Fluor 594-conjugated donkey anti-goat secondary antibodies (Invitrogen, A-11058).

\section{Immunofluorescence}

Cells were grown on coverslips and fixed in $4 \%$ formaldehyde (Electron Microscopy Sciences) diluted in PBS for $15 \mathrm{~min}$ at room temperature. Slides were washed 3 times with PBS and permeabilized for $20 \mathrm{~min}$ in blocking buffer containing $0.1 \%$ saponin (Sigma Aldrich, St Louis), $0.02 \%$ sodium azide, and $3 \%$ bovine serum albumin (BSA) in PBS. Primary and secondary antibodies were incubated in blocking buffer for $1 \mathrm{~h}$ at room temperature. Slides were washed in blocking buffer and PBS after primary and secondary antibodies. Coverslips were then mounted with the Prolong Gold antifade reagent with DAPI (Invitrogen). Fluorescent images were examined and photographed using confocal microscopy (Olympus FV10i, Tyoko, Japan).

\section{Chromatin immunoprecipitation}

Cells were cultured and subsequently crosslinked in $1 \%$ formaldehyde (Thermo Scientific, Carlsbad, CA, 28906) and processed according to the protocol from the Pierce $^{\mathrm{Tm}}$ Agarose ChIP Kit (Thermo Scientific, 26,156). Essentially, cells were lysed with Buffer 1 and pelleted. Pellets were resuspended in Buffer 1 and homogenized on ice and pelleted. Next, pellet was resuspended in Buffer 2 . The chromatin fraction was sheared by sonication in $1.5 \mathrm{ml}$ siliconized micro-centrifuge tubes. A $10-\mu \mathrm{g}$ aliquot of DNA was reverse crosslinked to assess chromatin size. $500 \mu \mathrm{g}$ of the resulting sheared chromatin samples were cleared overnight at $4{ }^{\circ} \mathrm{C}$. Also, TFE3 $(10 \mu \mathrm{g} / \mathrm{sample})$ and nonspecific goat IgG antibody (for background control) were incubated with magnetic beads overnight and washed with Buffer 3. Pre-cleared chromatin samples were added to washed beads and incubated at $4{ }^{\circ} \mathrm{C}$ overnight. Beads were washed with Buffer 3 and then eluted and reversed crosslink with Buffer 4 at $65{ }^{\circ} \mathrm{C}$ overnight. The promoter region of human 4EBP3 was amplified from immunoprecipitated genomic DNA with primers for amplification of -522 to -352 region: forward CTTAGCCTCCCAAAGTGCTG and reverse GCCAAAGTCACACATCTTGC; primers for amplification of -129 to +27 region: forward GGCT GGCTTCCTAGCAGATA and reverse GGCGTTGAG GTCGAGGAG; and primers for amplification of + 449 to + 639 region: forward GGCTGGCTTCCTAGCAGAT A and reverse GGCGTTGAGGTCGAGGAG.

\section{ChIP-seq analyses}

ChIP was performed in UOK109 $\left(3 \times 10^{8}\right)$ and UOK120 $\left(3 \times 10^{8}\right)$ cells using the TFE3 antibody. ChIP-seq data were obtained using an Illumina Hiseq 2000 sequencer (Illumina, San Diego, CA) and sequencing reads were de-multiplexed by Illumina pipeline [21]. ChIP-seq data were mapped to the human genome (hg19) using Bowtie algorithm with up to 2 mismatch allowed and reads mapping to more than 20 locations along the genome were discard. ChIP-seq data generated form IgG were used against the sample data in calling enriched regions and to control for false discovery rate (FDR). Peaks were called using MACS version 2, with q-value to 0.05 [22]. Gene ontology (GO) terms were categorized based on cell localization and the biological process. The $P$ values were obtained using DAVID Bioinformatics Resources 6.7. E-box sequence and distance from transcription start sites were analyzed using UCSC Genome Bioinformatics software.

\section{Luciferase reporter assay}

The 4EBP3 promoter was obtained from the GenBank and was cloned into the pGL4.10 vector (Obio, Shanghai, China, H6119). Cells were transfected with a firefly luciferase-containing reporter plasmid (4EBP3 promoter) and Renilla luciferase-containing plasmid as an internal control. Relative activity of firefly luciferase to Renilla luciferase was determined using the Dual-Glo luciferase assay system. Cells were seeded in 24-well plates followed by transfection with $0.1 \mu \mathrm{g} /$ well using Lipofectamine 2000 (Invitrogen). 


\section{Western blots}

Total protein was isolated from cells following various treatments. Cells were washed three times with PBS and lysed in ice cold extraction buffer $(50 \mathrm{mM}$ Tris- $\mathrm{HCl} \mathrm{pH} 7.4,150 \mathrm{mM} \mathrm{NaCl}, 1 \% \mathrm{NP}, 0.1 \%$ SDS, and $1 \times$ protease inhibitor cocktail). Whole cell lysates were centrifuged at $12000 \mathrm{~g}$ at $4{ }^{\circ} \mathrm{C}$. Soluble fractions were mixed with $5 \times$ loading buffer and heated at 100 ${ }^{\circ} \mathrm{C}$ for $5 \mathrm{~min}$. Protein concentration was determined by the Bradford method. Proteins were separated using SDS-PAGE and the PVDF membrane (Roche, Basel, Switzerland) by standard procedures. Blots were blocked for $1 \mathrm{~h}$ at room temperature in TBS with 0.05\% Tween 20 (Sigma Aldrich) and 5\% nonfat milk. Primary antibodies were incubated overnight at $4{ }^{\circ} \mathrm{C}$ in Tris Buffered Saline Tween (TBST) with 3\% BSA (Sigma Aldrich). HRP-conjugated secondary antibodies were incubated $1 \mathrm{~h}$ at room temperature. Blots were washed with TBST 6 times, 10 min each, after both primary and secondary antibody incubation. Protein bands were visualized with an enhanced chemiluminescence detection kit and recorded on a radiographic film (Alpha Innotech, San Jose, CA).

\section{Gene knockdown}

Cells were plated in 6-well plates and transfected with short hairpin RNA (shRNA) for human TFE3 (Obio, Y3619 and Y3620) and a non-target shRNA control (Obio, Y007) at a final concentration of $1 \mu \mathrm{g} /$ well using Lipofectamine 2000 Transfection Regent (Invitrogen) followed by incubation for $48 \mathrm{~h}$. Next, cells were passaged and analyzed for knockdown efficiency $72 \mathrm{~h}$ post-transfection. For viral infection, cells were cultured in 12-well plates and transfected with the TFE3 knockdown virus (Genechem, Shanghai, China, 54,157, 54,158 and 54,159) or a negative control virus (Genechem, CON077) using polybrene. $72 \mathrm{~h}$ after transfection, cells were infected at a MOI (multiplicity of infection) of 5 per well in 6-well plates. Puromycin was used to establish a stable cell line.

\section{RNA isolation and relative quantitative PCR}

Total RNA was isolated from cells using Trizol reagent (Invitrogen) according to the manufacturer's instruction. RNA was reverse-transcribed using Hiscript Q RT Supermix for qPCR (Vazyme, Nanjing, China, R122-01). RNA expression was quantified using the SYBR Green ER (Roche, Basel, Switzerland) according to the manufacturer's instruction. Duplicate total RNA samples were prepared from control and various treated cells and analyzed in triplicate by real-time PCR analysis using a 7300 system (Applied Biosystems, Grand Island, NY). The Ct values were analyzed using the $2^{-\Delta \Delta \mathrm{Ct}}$ method. Amplification of the reference endogenous gene GAPDH was used to normalize the sequence of interest. The primers for mRNAs were shown in Additional file 1: Supplement S1.

\section{Statistical analysis}

All calculations and statistical analyses were performed using SPSS for windows version 13.0 (SPSS Inc., Chicago, IL, USA). One-way analysis of variance (ANOVA) was used to analyze the differences between groups, followed by Dunnett's t-test. $P<0.05$ was regarded as statistically significant.

\section{Results}

TFE3 fusions accumulate in the nucleus in Xp11.2 tRCCs

Given that the intracellular distribution of TFE3 determines whether it can play a role in regulating cell metabolism as a transcription factor, we first aimed to investigate the intracellular distribution of TFE3 fusions. We found that TFE3 fusions accumulated in the nucleus in Xp11.2 RCC tumor samples (Fig. 1a), in contrast to few case of TFE3 localization within the nucleus in other renal clear cell cancer samples (Fig. 1b). These findings suggested that, once the TFE3 fused with other genes, it was mainly accumulated in the nucleus instead of the cytosol, which was further confirmed in UOK109 and UOK120 cells (Fig. 2a and b).

For UOK109 and UOK120 cells, we tested their fusion type, and found that UOK109 was NonO-TFE3 and UOK120 was PRCC-TFE3 (Additional file 2: Supplement $\mathrm{S} 2$ ), which was consistent with previous reports. Compared with none TFE3 fusion cells (786-0 and HK-2), the expression of fusion TFE3 genes increased significantly in UOK109 and UOK120 at both mRNA and protein levels (Fig. 2c and d).

According to the literature, active mTORC1 can phosphorylate TFE3 on Ser321, resulting in the creation of a binding site for the 14-3-3 proteins that TFE3 is sequestered in the cytosol without its transcriptional activity. To study the subcellular localization of the fusion TFE3 protein and its response to the mTORC1 signaling pathway, we introduced the mTOR inhibitor (PP242) into UOK109 and UOK120. After incubation with PP242 for 24h, TFE3 fusions showed no nuclear translocation whereas nuclear retention was observed in the control cells left untreated (Fig. 2a and b). In contrast, in 786-O and HK-2 cells, wild-type TFE3 proteins showed a rapid translocation from cytosol to the nucleus following by treatment with PP242 (Additional file 2: Supplement S2).

\section{TFE3 fusions escape from the control of mTOR signaling pathway}

TFE3 fusions fail to co-localize with LAMP2 in cytoplasm

In the cytoplasm, phosphorylated TFE3 can be recruited to the lysosomal surface through direct interaction with 


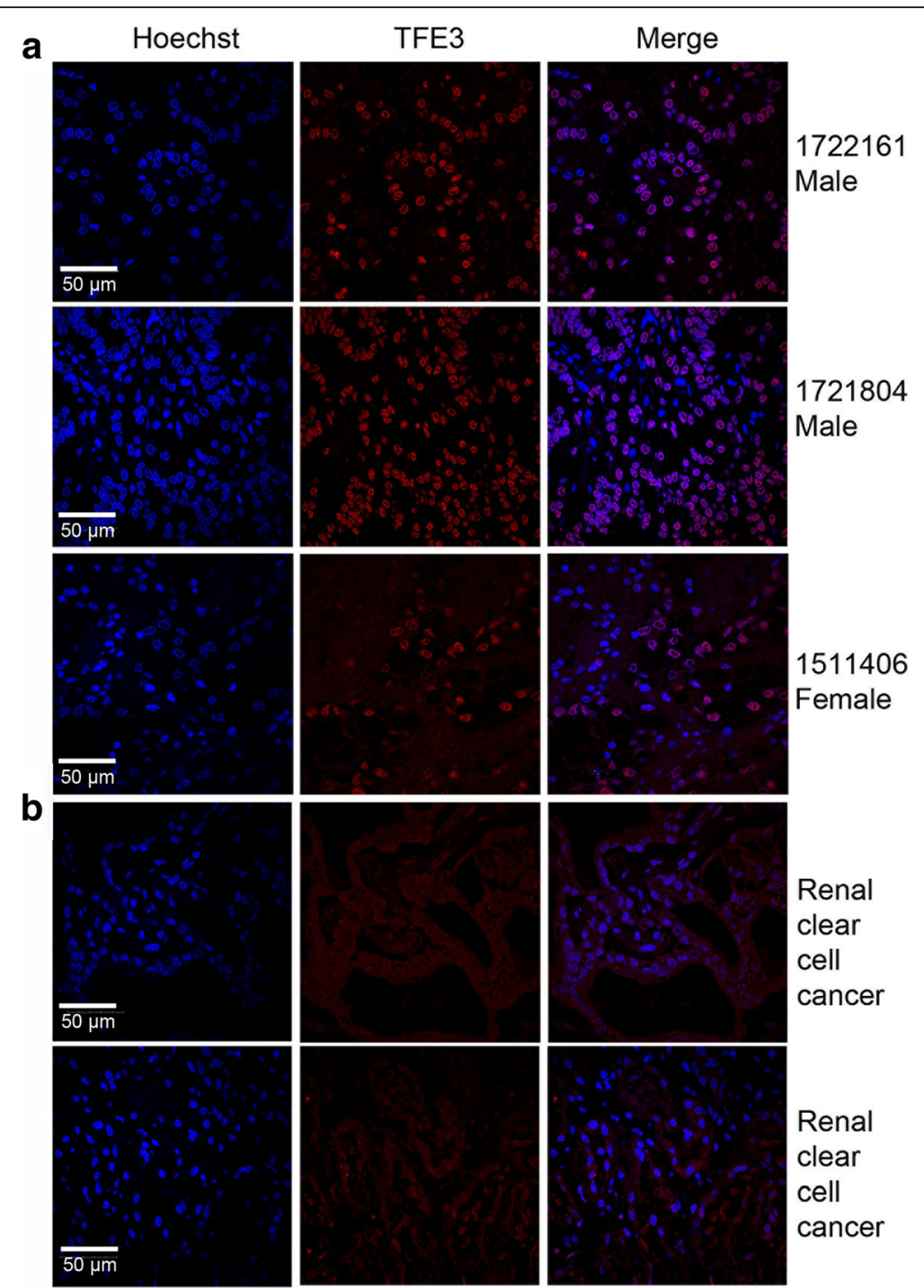

Fig. 1 TFE3 fusion proteins are accumulated in the nucleus in Xp11.2 translocation renal cell carcinoma (tRCCs). a, b TFE3 proteins (red) were measured with immunofluorescence microscopy in the Xp11.2 tRCCs pathological sections and the renal clear cell carcinoma pathological sections. Nuclei were stained with Hoechst (blue). Scale bar, $50 \mu \mathrm{m}$

active mTORC1 and this recruitment is critical for maintaining TFE3 sequestration in the cytosol. LAMP2 (lysosome-associated membrane protein 2) is a lysosomal surface marker. We found that wild-type TFE3 could co-localize with LAMP2, and incubation with a mTOR inhibitor (PP242 or Torin1) could separate LAMP2 from TFE3 in HK-2 (Fig. 3a and d). However, TFE3 fusions showed rare co-localization with LAMP2 in UOK109 and UOK120 cells, in which TFE3 fusions were mostly located in the nucleus and LAMP2 stayed at cytoplasm (Fig. 3b, c, e, f). And TFE3 fusions remained its nuclear retention after treatment with mTOR inhibitor. These data indicated that the subcellular localization of TFE3 fusions was capable of escaping from the control of mTOR signaling pathway and could not be recruited to the lysosomal surface.

\section{Interaction between TFE3 fusions and the cytosolic chaperone 14-3-3 is decreased in UOK109 and UOK120 cells}

The 14-3-3 proteins have been identified as major binding partners of TFE3. The 14-3-3 proteins typically interact with their targets via short phosphoserine containing motifs. In order to determine whether 14-3-3 proteins were implicated in the control of TFE3 subcellular localization, we screened for candidates through the whole family of cytosolic chaperone 14-3-3 proteins. Three subtypes of $14-3-3$ proteins, including $14-3-3 \alpha$, 

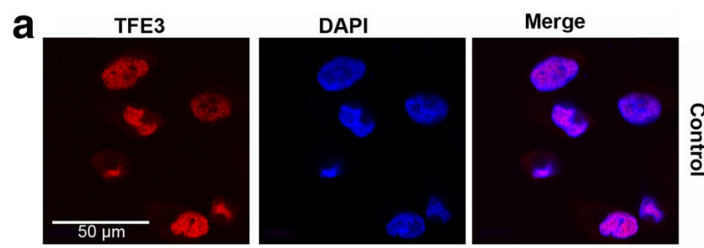

\section{C}
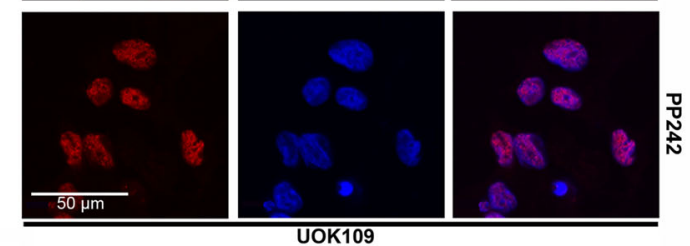

\section{§ิ}

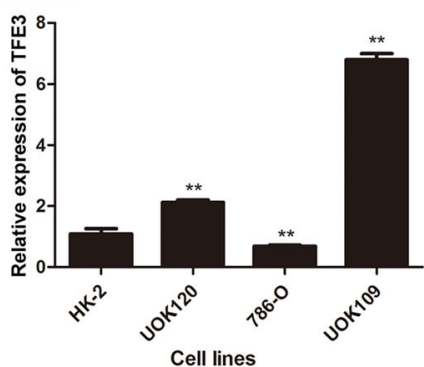

d
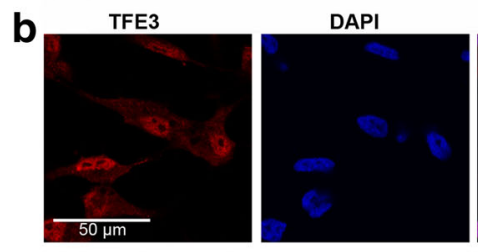

Merge
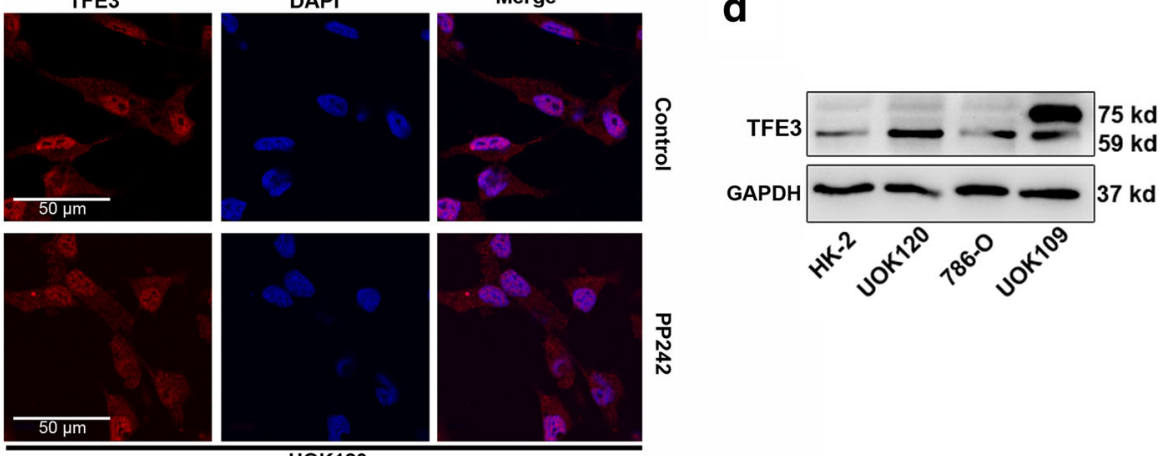

勇

UOK120

Fig. 2 PP242 exerts no effect on the subcellular localization of TFE3 fusion proteins. Renal carcinoma cell lines including HK2, UOK109 and

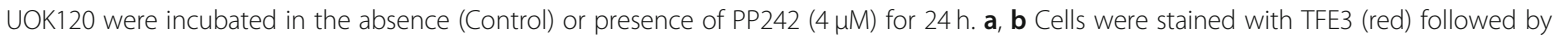
immunofluorescence photomicrographic analysis. $\mathbf{c}$, $\mathbf{d}$ The expression of TFE3 was determined by q-PCR and Western blot. GAPDH was used as an internal control. ${ }^{*} P<0.05,{ }^{* *} P<0.01$. Scale bar, $50 \mu \mathrm{m}$

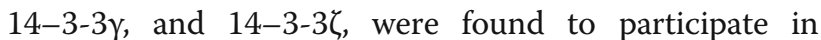
TFE3 cytosol retention (Fig. 4a). Although the other three isoforms of the 14-3-3 proteins were also changed, multiple protein bands were revealed which implied that they could interact with other isoforms to form heterodimers. As shown in Fig. $4 a$ and b, the $14-3-3 \gamma$ was decreased apparently in 786-0 and HK-2 cells after treatment with the mTOR inhibitor. While there were no differences in UOK109 and UOK120 irrespective of treatment with the mTOR inhibitor (Fig. 4c-f). The Co-IP data suggested that the binding between TFE3 fusions and 14$3-3 \gamma$ was reduced in UOK109 and UOK120 cells compared with in HK-2 cells (Fig. 4g). Together, these data indicated that TFE3 fusions failed to bind with the cytosolic chaperone $14-3-3 \gamma$ which was able to induce the nuclear accumulation of TFE3 fusions.

\section{Fusion segment of TFE3 proteins cannot translocate to nucleus}

According to previous studies, the first $30 \mathrm{~N}$-terminal residues of TFE3 are both necessary and sufficient for interaction with active Rag heterodimers which could bind the mTORC1 and recruit TFE3 to lysosomes [10]. The TFE3 fusion fragment in UOK109 were 296-575 amino acid longs at C-terminal and the fusion fragment in UOK120 was composed of the 179-575 amino acids at C-terminal. Based on these findings, we designed three types of expression plasmids containing TFE3 fused to EGFP including H8116 (TFE3 296-575aa), 40,770 (TFE3 179-575aa) or H11660 (full TFE3). The full TFE3 was used as a positive control. Western blot revealed that the TFE3 was dramatically increased in H11660 infected group and its target protein 4EBP3 was increased at the same time (Fig. 5a). However, in H8116 and 40,770 infected group, even TFE3 (296-575 aa) and TFE3 (179-575 aa) showed evidently increases. In contrast, the expression of 4EBP3 was not increased (Fig. 5a). TFE3 (296-575 aa) and TFE3 (176-575 aa) were mainly increased in the nucleus. Few of TFE3 protein segments were expressed in the cytoplasm as confirmed by Western blot (Fig. 5b). TFE3 (296-575 aa) and TFE3 (176-575 aa) were mainly localized in the cytoplasm together with EGFP in 293 T cells confirmed by immunofluorescence assays. Few TFE3 segments were localized in the nucleus (Fig. 5c and d). Whereas, the full TFE3 protein was localized both in the cytoplasm and the nucleus (Fig. 5e). All these data demonstrated that TFE3 fusion segments only expressed and were localized in the cytoplasm but not in the nucleus. 

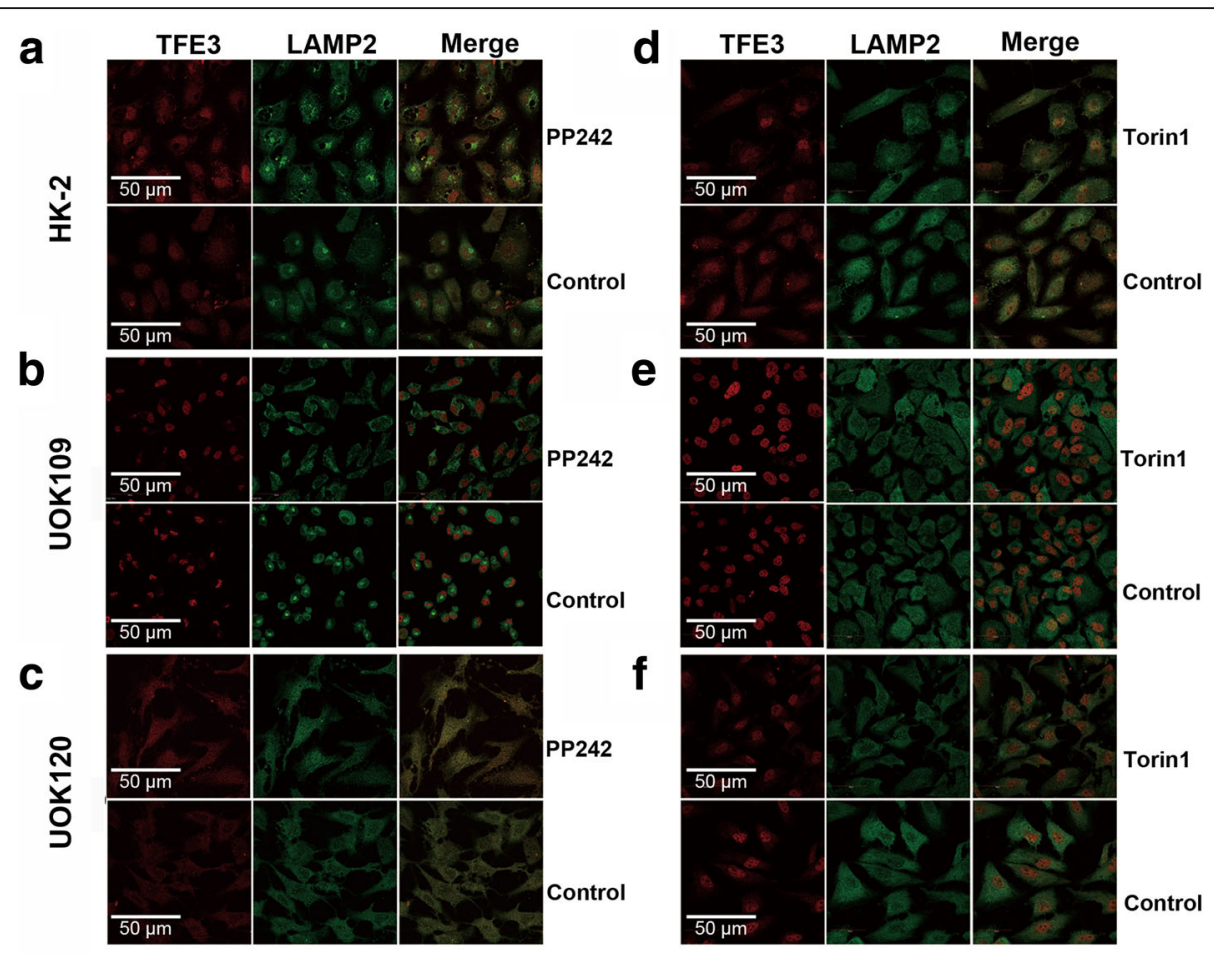

e

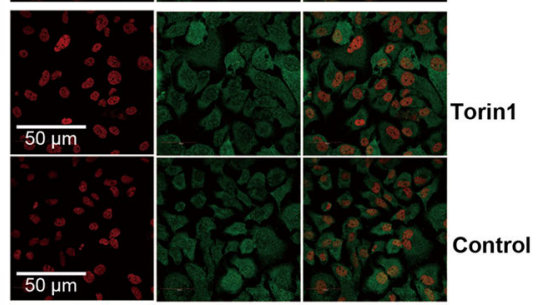

f
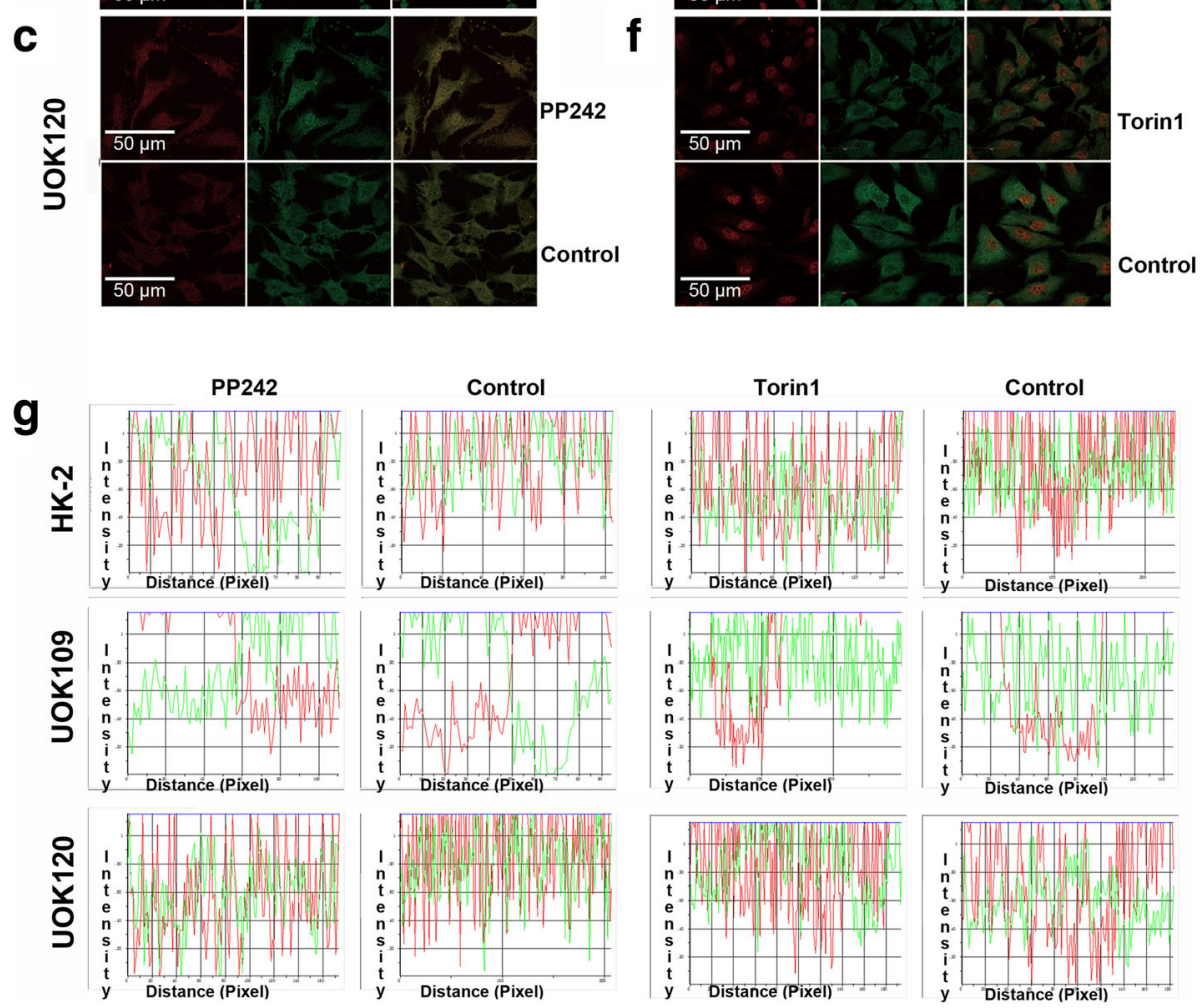

Fig. 3 TFE3 fusion proteins fail to co-localize with the lysosomal protein LAMP2. Renal carcinoma cell lines including HK2, UOK109 and UOK120 were treated with PP242 $(4 \mu \mathrm{M})$ or torin1 $(4 \mathrm{nM})$ or left untreated (Control). a to $\mathbf{f}$ Cells were stained with antibodies against TFE3 (red) and LAMP (green) followed by immunofluorescence photomicrographic analysis. (g) The merging of the red and green fluorescence signals shown in a to $f$ were quantified with Image-pro Plus. Scale bar, $50 \mu$

\section{Inhibition of GSK3 $\beta$ increases the cytoplasm retention of TFE3 fusions}

Two residues (Ser134 and Ser138) in TFE3 are highly conserved in MiTF family, which could be phosphorylated by GSK3 $\beta$ leading to their cytoplasmic retention [23, 24]. In UOK109 and UOK120 cells, we found that only PRCC-TFE3 fusions retained the two residues by sequence alignment (Additional file 3: Supplement S3). Our data showed that TFE3 fusions failed to be subject to the regulation by mTORC1 and accumulated in the nucleus in Xp11.2 tRCCs. In order to increase cytoplasmic retention of TFE3 fusions, we activated GSK3 $\beta$ through inhibiting AKT using MK2206 in UOK120 cells. It was revealed that TFE3 fusions were evidently increased in the cytoplasm (Fig. 6a). In addition, the uncoupled TFE3 in the cytoplasm could be dephosphorylated and degraded through ubiquitination. Next, we inhibited the ubiquitination degradation pathway by MG132 in HK-2 cells. The 

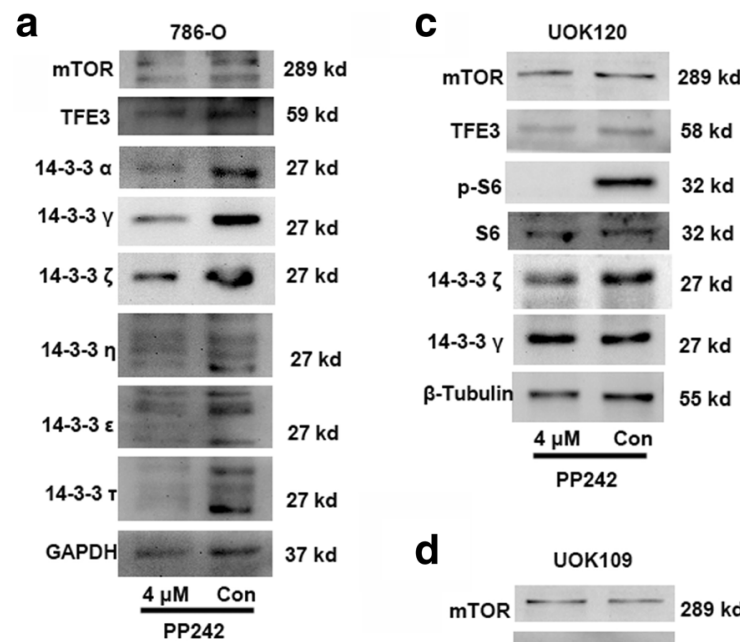

e
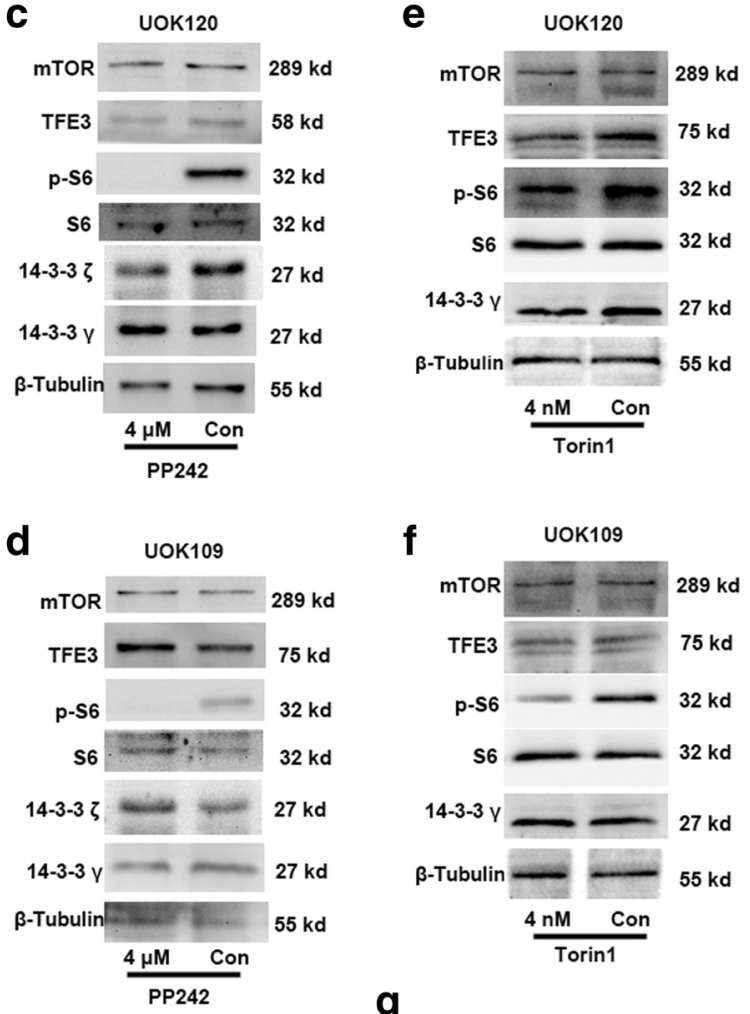

f
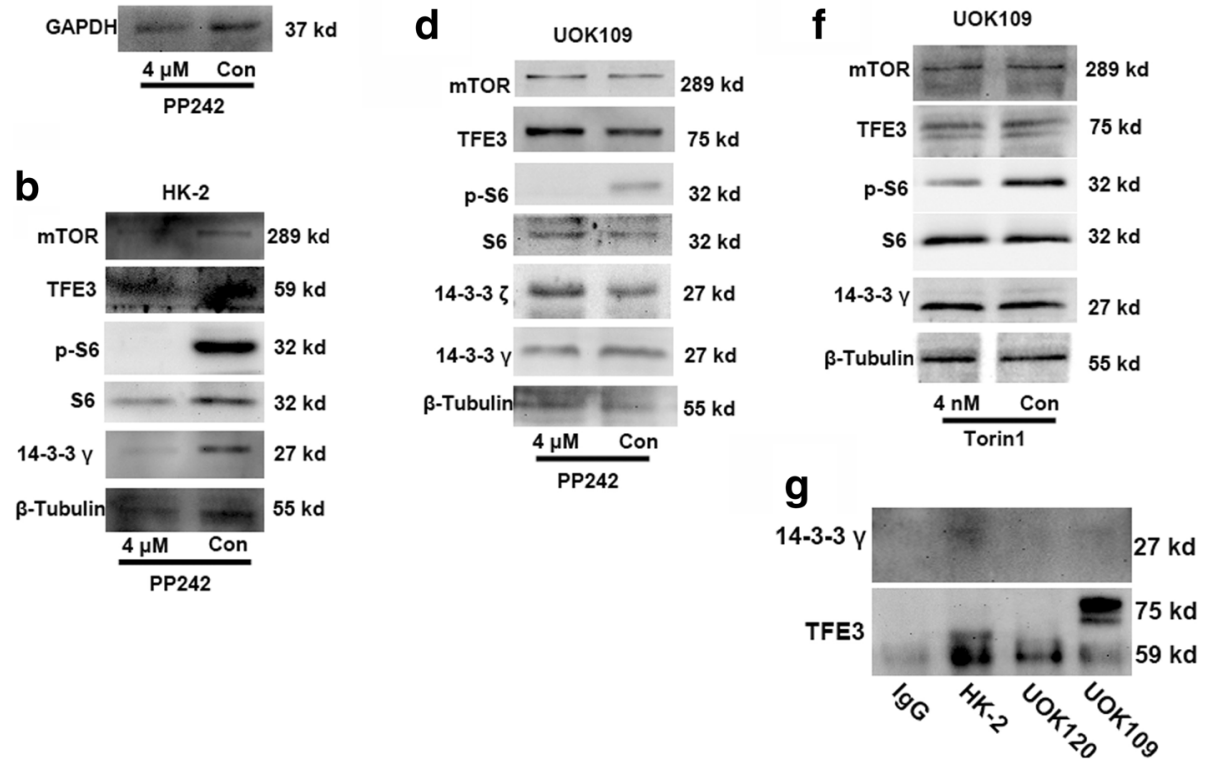

Fig. 4 Binding between 14 and 3-3 proteins and TFE3 fusion proteins is reduced in the UOK109 and UOK120 cells. (a) 786-O cells were treated with PP242 $(4 \mu \mathrm{M})$ or vehicle control (Con) for $24 \mathrm{~h}$ followed by immunoblot analysis of 14-3-3 proteins. GAPDH was used as an internal control.

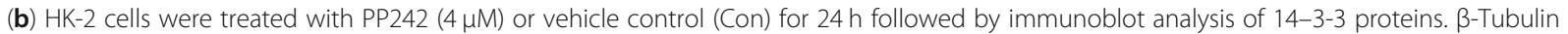

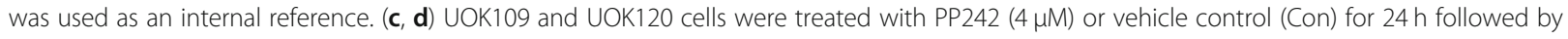
immunoblot analysis of 14-3-3 $\gamma, 14-3-3 \zeta, \mathrm{p}-\mathrm{S} 6, \mathrm{~S} 6$ and mTOR proteins. (e, f) HK-2 cells were treated with Torin1 (4 nM) or vehicle control (Con) for $24 \mathrm{~h}$ followed by immunoblot analysis of 14-3-3 $\gamma$, phosphor-S6 (p-S6), S6 and mTOR proteins. (g) The binding between TFE3 and 14-3-3 $\gamma$ was detected by co-immunoprecipitation (CO-IP). An irrelevant IgG was used as negative control

expression of TFE3 was increased and mTOR inhibition mediated reduction was recovered after treatment with MG132 (Fig. 6b and c). These data indicated that activating Gsk3 $\beta$ could lead to retention of TFE3 fusions in the cytoplasm which was accompanied with a reduction in its nuclear retention.

\section{TFE3 fusions bind to the promoters of targeted-genes as native TFE3}

To explore the molecular function of TFE3 fusions, we performed chromatin immunoprecipitation coupled to deep sequencing (ChIP-seq). We identified 4219 peaks in UOK109 cells and 1584 peaks in UOK120 cells (Additional file 4: Supplement S4). The predicted target genes in UOK109 and UOK120 cells were shown in Additional file 5: Supplement S5 and Additional file 6: Supplement S6. In order to gain an insight into the identification of the promoters occupied by TFE3 fusions, we used the web tool DAVID to perform GO analysis. Components associated with localization, organic substance transport, lytic vacuole organization, lysosome organization, and transport as shown in Table 1 significantly interacted with TFE3 fusions in UOK109 cells. The analysis of lysosomes demonstrated significant increases of the number of genes involved in the lysosome organization, endosome to lysosome transport and phagolysosome assembly, which suggested that TFE3 fusions could bind to the CLEAR element and regulate lysosomal biosynthesis. The analysis of KEGG pathway revealed that lysosomes were correlated with TFE3 (Table 2) which further confirmed 


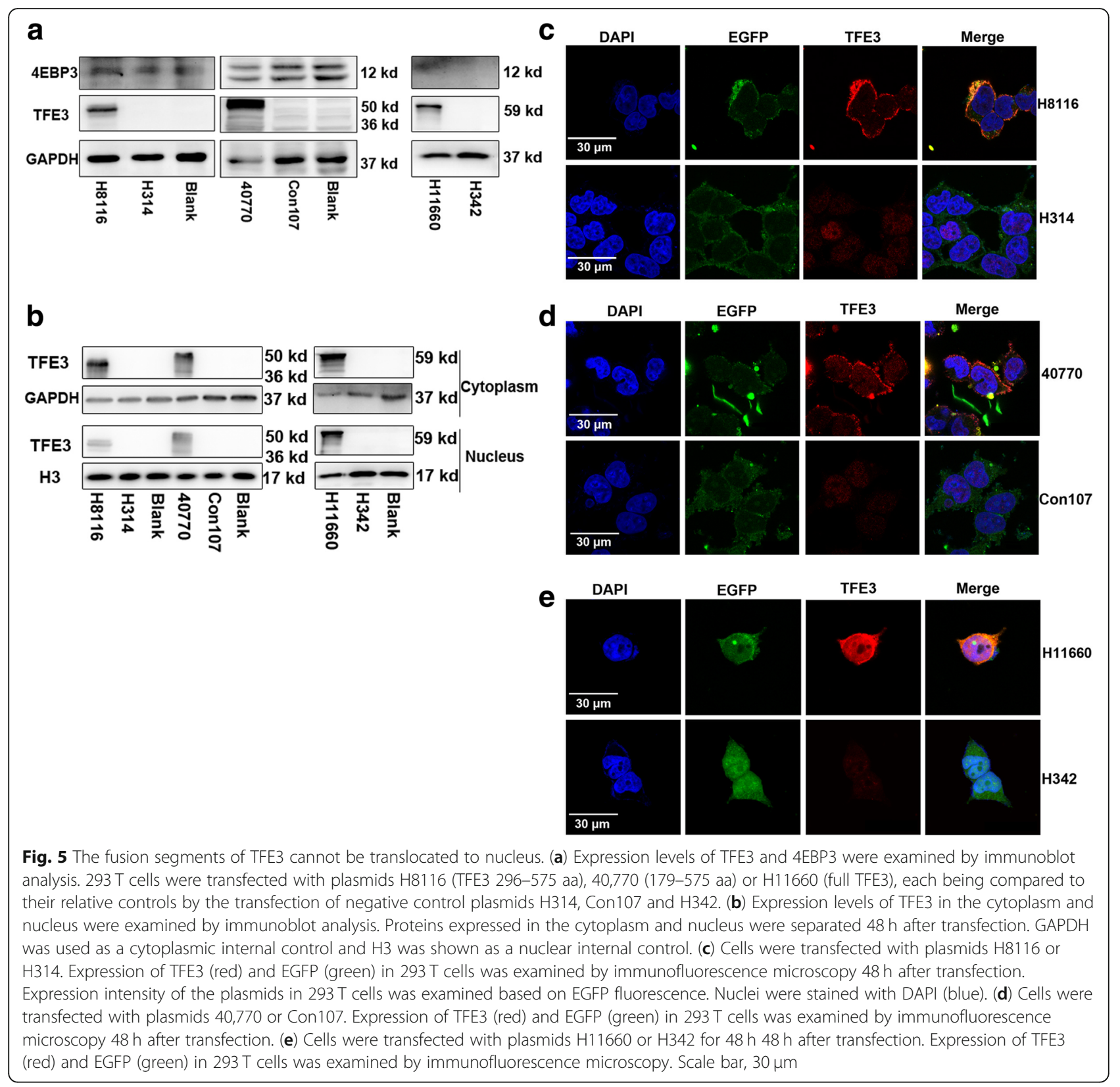

NonO-TFE3 fusions could still regulate lysosomal biogenesis genes as the wild-type TFE3. GO analysis in the UOK120 cells showed that components associated with the regulation of cellular macromolecule biosynthetic processes were significantly correlated with TFE3 fusions (Table 3). Different from UOK109 cells, the predicted gene functions mainly focused on the transcription processes and nutrient metabolic processes but not the lysosomal biogenesis in UOK120 cells. Furthermore, the MAPK signaling pathway and focal adhesion pathway showed significant correlation with PRCC-TFE3 fusions by KEGG pathway analyses (Table 4 ).
Since TFE3 fusions were significantly overexpressed and accumulated in the nucleus in UOK109 and UOK120 cells, we found that TFE3 fusions might also influence the expression of lysosomal genes or other target genes. In UOK109 and UOK120 cells, down-regulation of TFE3 fusions decreased the mRNA abundance of 8 out of 10 genes tested, including 4EBP3, SQSTM1, VPS8, VPS11, CCND3, ATP5G, ATP5O and GOLPH3. The expression of 4EBP3 at the protein level showed a significant decrease after TFE3 knockdown with plasmids or viral infection (Fig. 7a-d). In addition, the expression of S6 and p-S6 which are downstream of 


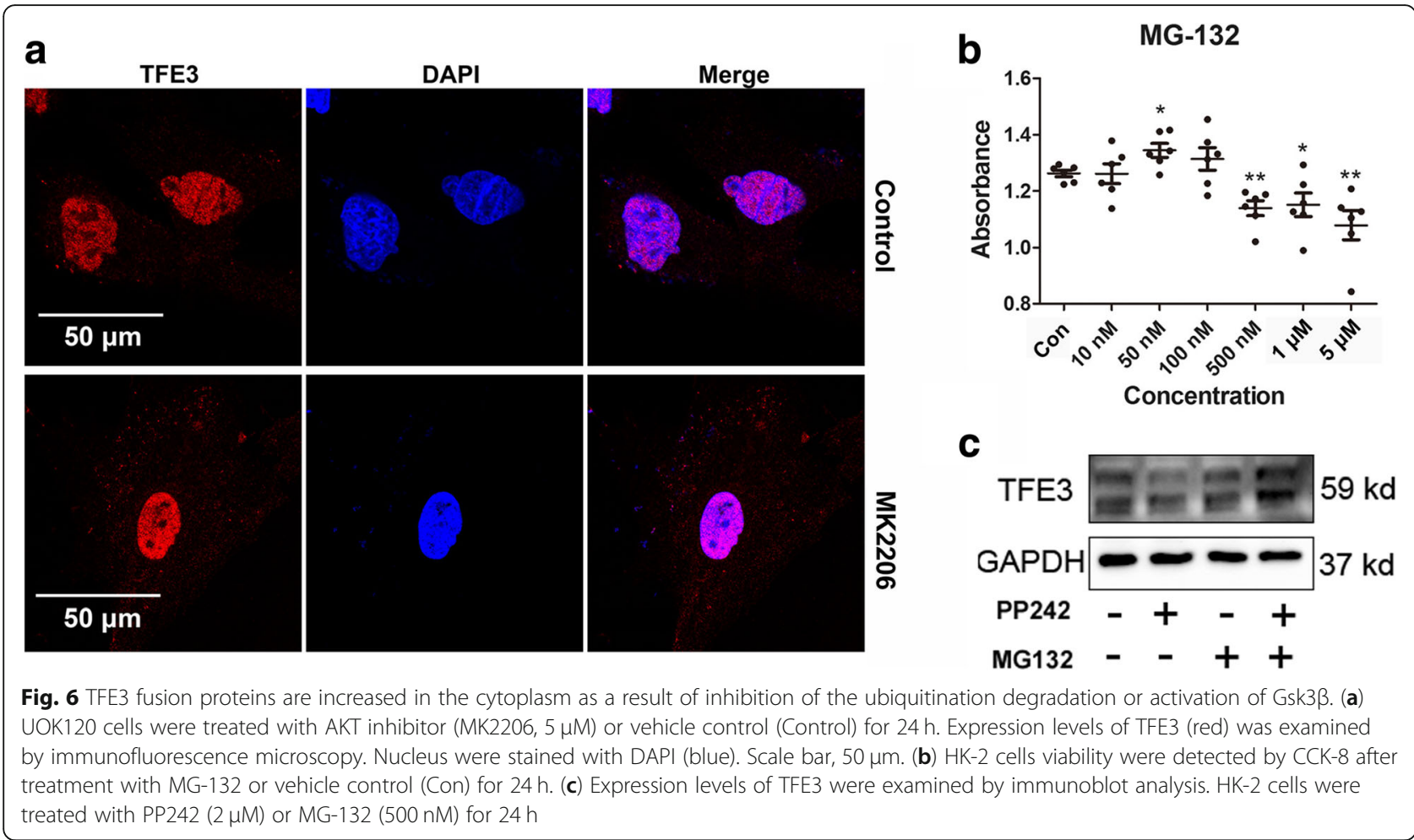

mTORC1 also showed an evident decrease. In TFE3 knockdown cells, the expression of TFE3 fusions was reduced, while the subcellular localization of TFE3 fusions remained unchanged in UOK109 and UOK120 cells (Fig. 7e and f). These data suggested that TFE3 fusions could bind to the CLEAR element as the wild-type TFE3 and could regulate the expression of lysosomal biogenesis genes.

Furthermore, we chose 4EBP3 as a target gene to test the transcriptional activity of TFE3 fusions. To examine how the transcriptional induction of 4EBP3 was regulated by TFE3 fusions, we generated luciferase reporter plasmids containing the 4EBP3 promoter region. Luciferase assay data showed that TFE3 fusions could bind to

Table 1 The GO analysis data of the target genes in UOK109

\begin{tabular}{lll}
\hline GOBPID & Term & Pvalue \\
\hline GO:0051179 & localization & $2.02 \mathrm{E}-05$ \\
GO:0071702 & organic substance transport & $4.68 \mathrm{E}-05$ \\
GO:0007040 & lysosome organization & $8.18 \mathrm{E}-05$ \\
GO:0080171 & lytic vacuole organization & $8.18 \mathrm{E}-05$ \\
GO:0006810 & transport & $9.80 \mathrm{E}-05$ \\
GO:0051234 & establishment of localization & 0.000125094 \\
GO:0071705 & nitrogen compound transport & 0.000309726 \\
GO:0070482 & response to oxygen levels & 0.000391147 \\
GO:0061025 & membrane fusion & 0.000394916 \\
GO:0006687 & glycosphingolipid metabolic process & 0.000417151 \\
\hline
\end{tabular}

the 4EBP3 promoter region and activate its transcription, which indicated that the TFE3 fusions remained its transcriptional activity (Fig. 8a and b). In addition, we performed a chromatin immunoprecipitation assay to determine whether TFE3 fusions directly binds to the 4EBP3 promoter at the E-box. The chromatin immunoprecipitation data showed that TFE3 fusions bind to the DNA segment containing the E-box $(-129$ to +27$)$ of the 4EBP3 promoter, but not the sites from -522 to 352 or from +449 to +639 (Fig. $8 \mathrm{c}$ and d). Taken together, these results demonstrated that TFE3 fusions could mediate the transcriptional initiation of target genes just like the wild-type TFE3.

Table 2 The KEGG pathway analysis of the target genes in UOK109

\begin{tabular}{lll}
\hline KEGGID & Term & Pvalue \\
\hline 4142 & Lysosome & $3.91 \mathrm{E}-06$ \\
604 & Glycosphingolipid biosynthesis - ganglio series & 0.002229366 \\
520 & Amino sugar and nucleotide sugar metabolism & 0.002417655 \\
760 & Nicotinate and nicotinamide metabolism & 0.013163582 \\
5144 & Malaria & 0.013980843 \\
524 & Butirosin and neomycin biosynthesis & 0.014139062 \\
603 & Glycosphingolipid biosynthesis - globo series & 0.015695803 \\
3440 & Homologous recombination & 0.022457622 \\
5216 & Thyroid cancer & 0.025272857 \\
511 & Other glycan degradation & 0.026898164 \\
\hline
\end{tabular}


Table 3 The GO analysis data of the target genes in UOK120

\begin{tabular}{lll}
\hline GOBPID & Term & Pvalue \\
\hline GO:2000112 & regulation of cellular macromolecule biosynthetic process & $6.88 \mathrm{E}-05$ \\
GO:0006355 & regulation of transcription, DNA-templated & $7.56 \mathrm{E}-05$ \\
GO:1903506 & regulation of nucleic acid-templated transcription & $8.70 \mathrm{E}-05$ \\
GO:2001141 & regulation of RNA biosynthetic process & 0.000100025 \\
GO:0009889 & regulation of biosynthetic process & 0.000130783 \\
GO:0010556 & regulation of macromolecule biosynthetic process & 0.000139177 \\
GO:0051252 & regulation of RNA metabolic process & 0.000192021 \\
GO:0006351 & transcription, DNA-templated & 0.000206295 \\
GO:0031326 & regulation of cellular biosynthetic process & 0.000207896 \\
GO:0097659 & nucleic acid-templated transcription & 0.000228788
\end{tabular}

It is reported that TFE3 mediates the transcriptional initiation of 4EBP3 in response to mTORC1 inhibition [25]. To study the function of 4EBP3, we examined the effect of mTOR inhibitors (PP242 and Torin1) on the expression of 4EBP3 in 786-0, HK-2, UOK109 and UOK120 cell lines. Prolonged treatment of renal cell lines with mTOR inhibitors resulted in an increase in 4EBP3 at the mRNA level (Fig. 9a-f). Furthermore, we found that fold-change of 4EBP3 in UOK109 and UOK120 were significantly reduced compared with HK-2 and 786-O cells (Fig. 9g). These findings suggested that TFE3 fusions promote the expression of 4EBP3 after mTOR inhibition, while the effect of mTOR inhibitor on TFE3 fusions was diminished in UOK109 and UOK120.

\section{Discussion}

Since the discovery of TFE3 gene fusions, the mechanism underlying the oncogenic effects of these mutations in kidney largely remains unknown. As with other fusion proteins involving transcription factors, promoter substitution appears to be the key molecular event with TFE3 fusions, causing abnormal TFE3 protein activity. All these fusion isoforms become fused with housekeeping genes containing stronger promoters than normal TFE3. In this study, we found that the expression of NonO-TFE3 and PRCC-TFE3 fusions were definitely increased in UOK109 and UOK120 cells compared with the HK-2. In addition, the overexpressed TFE3 fusions showed strong nuclear retention.

According to literature reports, subcellular localization of TFE3 affected its function as a transcription factor.

Table 4 The KEGG pathway analysis of the target genes in UOK120

\begin{tabular}{lll}
\hline KEGGID & Term & Pvalue \\
\hline 4010 & MAPK signaling pathway & 0.034063246 \\
4510 & Focal adhesion & 0.045985224 \\
\hline
\end{tabular}

And a recent study showed that stage-specific subcellular relocalization of TFE3 contributes to developmental progression of pluripotent epiblast [26]. TFE3 nuclear translocation is regulated by several signals including mTORC1, ER stress, mitochondrial damage, and pathogen infection $[27,28]$. In fully fed cells, TFE3 is recruited to lysosomes through phosphorylation at Ser321 by activating mTORC1 and is retained in the cytosol by the cytosolic chaperone 14-3-3 proteins. Following starvation, lysosomal stress and other conditions inhibit mTORC1, TFE3 is rapidly translocated to the nucleus and activates gene transcription. In contrast to previous studies, we found that TFE3 fusions were capable of escaping from the regulation of $\mathrm{mTORC} 1$. Our results revealed that TFE3 fusions had lost its cytosol retention and were mainly accumulated in the nucleus in UOK109 and UOK120 cells. In the cytoplasm, the interaction between TFE3 fusions and 14-3-3 proteins rarely occurred in UOK109 and UOK120 cells. We further demonstrated that TFE3 fusions failed to be localized on the lysosomal surface and were mainly localized in the nucleus. Besides, the TFE3 segments expressed in $293 \mathrm{~T}$ cells showed evident cytosolic retention, while the full TFE3 were localized both in the nucleus and the cytoplasm. Bioinformatics analysis revealed that the fused TFE3 gene of UOK109 cells contained 6-10 exons and the fusion TFE3 gene of UOK120 contained 4-10 exons [14]. The TFE3 exon 1 was invariably absent and the universally retained region of the TFE3 gene (6-10 exons) corresponded to a 280 aa C-terminal peptide, which includes the bHLH/LZ dimerization/DNA-binding domain and the putative nuclear localization signal (NLS). Within the fusion PRCC sequence in the UOK120 cells, another NLS can be identified (KPKKRK at position 137) at the N-terminal side of the fusion [29]. Consequently, PRCC-TFE3 contains both NLS sequence which can promote its translocation into the nucleus [30]. The NLS also existed in the N-terminal of the fusion $\mathrm{NonO}$ 


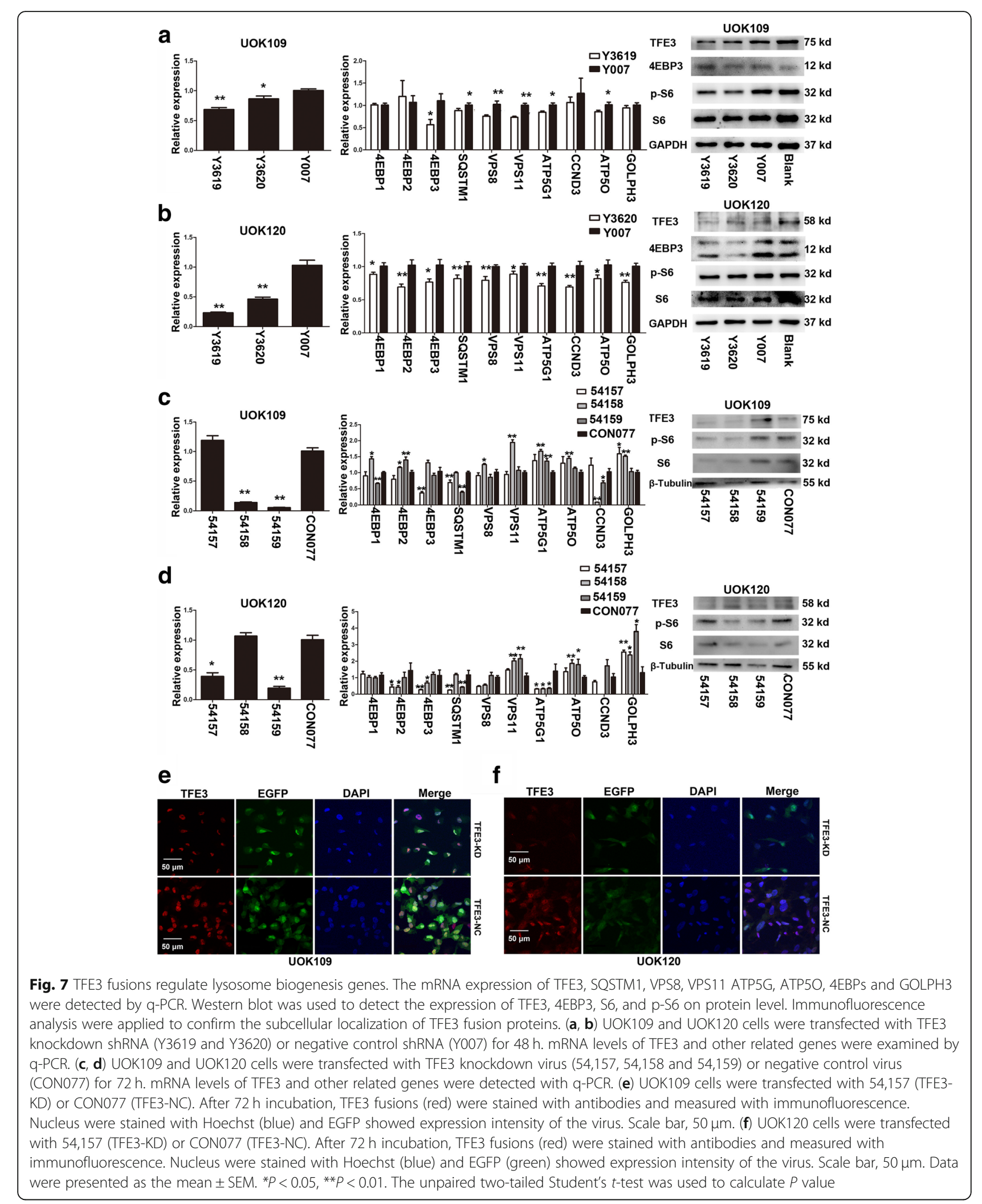




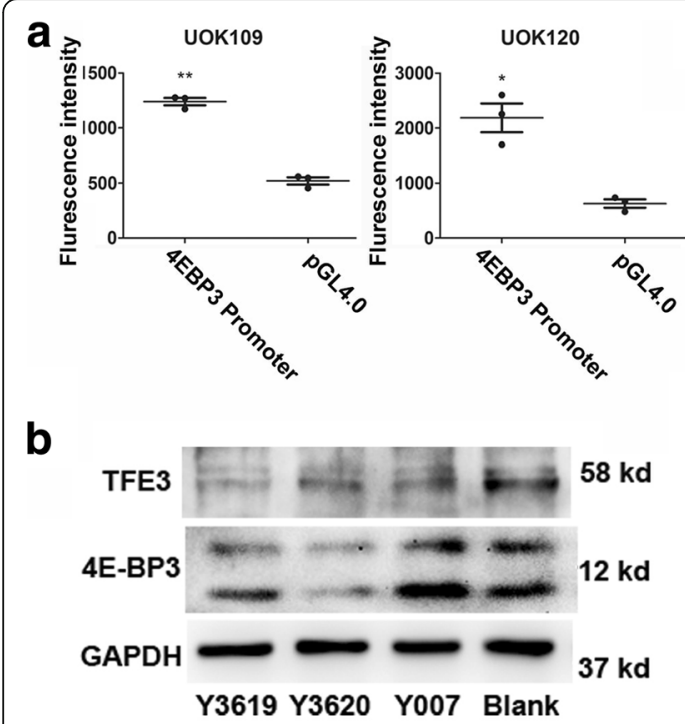

C

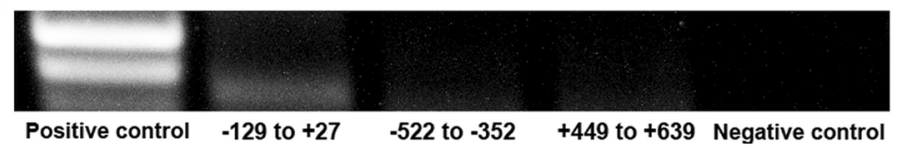

Fig. 8 TFE3 fusions could bind to the promoter of 4EBP3. (a) The transcriptional activity of TFE3 fusions with different splicing variants was detected with luciferase reporter assay in UOK109 and UOK120 cells. Cells were transfected with 4EBP3 promoter expressing plasmids (4EBP3 Promoter) or negative control plasmids (pGL4.0) for $48 \mathrm{~h}$. (b) Western blot was used to determine the expression of TFE3 and 4EBP3 after $48 \mathrm{~h}$ transfection of TFE3 knockdown shRNA (Y3169 and Y3620), negative control shRNA (Y007) or blank control (Blank). (c) The interaction between TFE3 fusions and 4EBP3 promoter was detected by Chromatin immunoprecipitation (ChIP). PCR was applied to detect the target DNA fragments. (d) The predicted target binding sites to $4 \mathrm{EBP} 3$ were showed in the table. Data were presented as the mean \pm SEM. ${ }^{*} P<0.05$, ${ }^{* *} P<0.01$. The unpaired two-tailed Student's $t$-test was used to calculate $P$ value

sequence in UOK109. The NonO-TFE3 results in fusion of almost the entire splicing factor protein to the C-terminal part of TFE3 containing the DNA-binding domain [3]. Therefore, the NonO-TFE3 contained one NLS which also could lead to strong nuclear translocation. In addition, the first $30 \mathrm{~N}$-terminal residues of TFE3, which were absent in the TFE3 fusion proteins, were both necessary and sufficient for interaction with active Rag heterodimers. Although the TFE3 fusions in UOK109 and UOK120 retained the phosphorylation site at Ser321, they lost the first 30 residues making them unavailable for the lysosome recruitment under mTORC1 inhibition. Taken together, the TFE3 fusion proteins could not be regulated by mTORC1 mainly as a results of losing the lysosomal localization signal. The nuclear retention of TFE3 fusions may be attributed to the retained NLS at the C-terminal of the fusion peptide.

The phosphorylation of multiple conserved amino acids mediates TFE3 subcellular localization and protein stabilization. The mTOR kinase was shown to phosphorylate specific serine residues in TFE3 and to play a major role in the regulation of TFE3 subcellular localization $[10,13]$. However, in our study the TFE3 fusions were not controlled by the mTOR signaling pathway and were completely accumulated in the nucleus. Therefore, we turned our attention to other phosphorylation sites on
TFE3. Previous studies indicated that TFEB can be phosphorylated by GSK3 $\beta$ at residues Ser134 and Ser138, which are highly conversed between TFE3 and TFEB, leading to cytoplasmic retention [31]. In UOK120 cells, phosphorylation of these residues through activation of GSK3 $\beta$ could distinctly increase TFE3 fusions in cytoplasm and decrease their nuclear retention. In addition, the dissociated TFE3 could be dephosphorylated by $\mathrm{PKC} \beta$ or $\mathrm{AKT}$ at $\mathrm{C}$-terminal in the cytoplasm leading to TFE3 ubiquitination and degradation [31-33]. In the HK-2 cells, inhibition of ubiquitination degradation increased TFE3 expression in the cytoplasm. Taken together, phosphorylation of TFE3 fusions by GSK3 $\beta$ promotes its cytoplasmic retention and dissociated TFE3 fusions in the cytoplasm can be degraded through the ubiquitination pathway. All these could provide potential therapeutic targets for decreasing the nuclear retention of TFE3 fusions in Xp11.2 tRCCs.

A recent study revealed that TFE3 can activate gene expression associated with autophagy and lysosomal biogenesis which promote cellular survival during starvation [10]. In this study, we demonstrated that the overexpressed TFE3 fusions could promote the expression of lysosomal biogenesis genes. Furthermore, the ChIP-seq analysis indicated that TFE3 fusions still maintained their regulation of lysosomal biogenesis in the UOK109 cells. Lysosomes are the primary degradation 

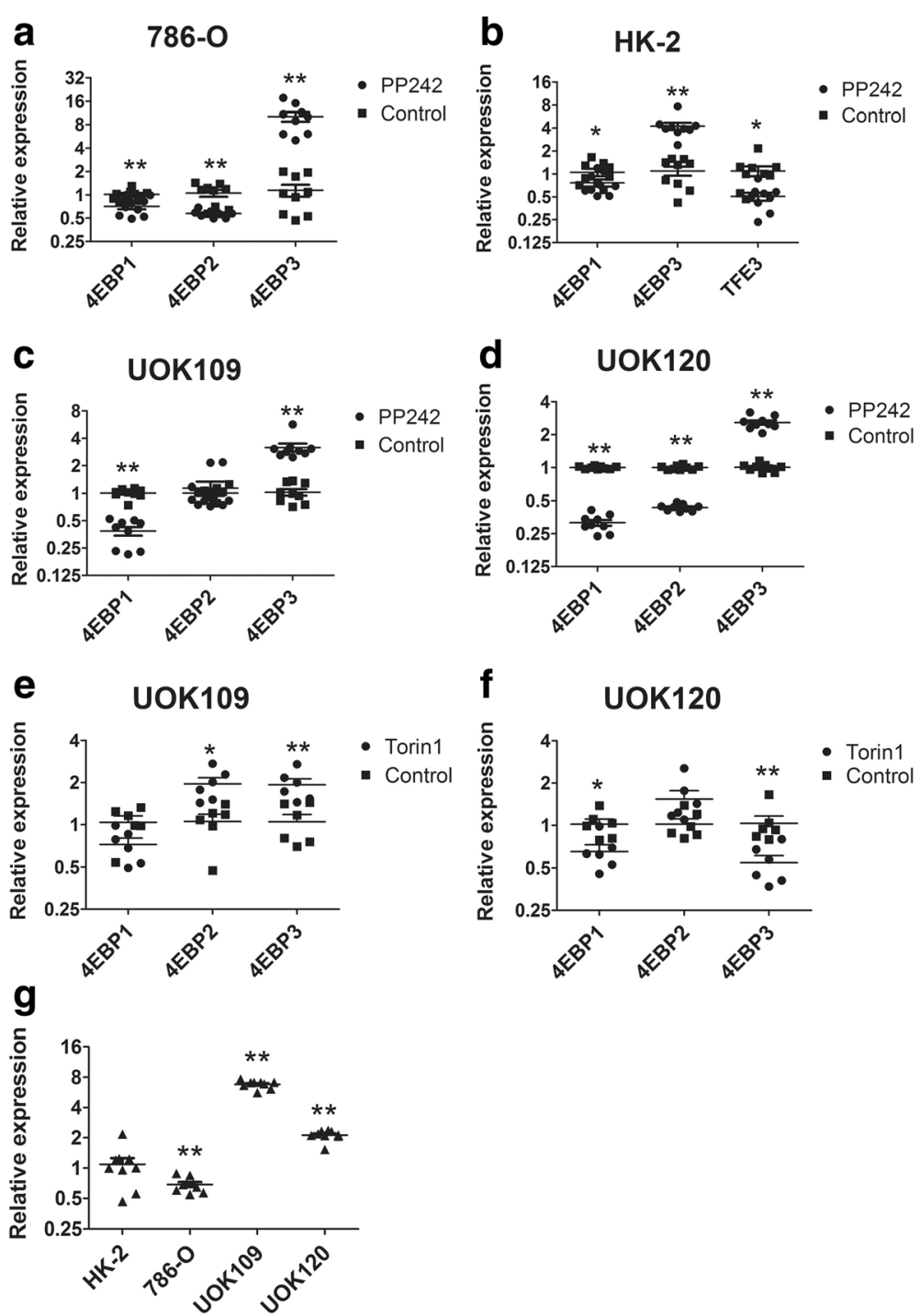

Fig. 9 TFE3 fusions promote the expression of 4EBP3 after mTORC1 inhibition. (a to d) 786-O, HK-2, UOK109 and UOK120 cells were treated with mTOR inhibitor (PP242) or DMSO (Control) for $24 \mathrm{~h}$. The expression of 4EBPs were determined by q-PCR, and GAPDH was used as an internal control. (e, $\mathbf{f}$ ) The expression of 4EBPs were detected by $\mathrm{q}-\mathrm{PCR}$ after $24 \mathrm{~h}$ treatment with mTOR inhibitor (Torin 1 ) or left untreated (Control) in UOK109 and UOK120 cells. (g) The fold-change of 4EBP3 in 786-O, UOK109 and UOK120 cells compared with HK-2. Data were presented as the mean \pm SEM. ${ }^{*} P<0.05$, ${ }^{*} P<0.01$. The unpaired two-tailed Student's $t$-test was used to calculate $P$ value

organelles in cells. Lysosomes receive extracellular materials destined for degradation through endocytosis, whereas intracellular components reach lysosomes mainly through autophagy [34]. The increase of lysosomes may lead to frequent biomolecule degradation and recycling. Thus, cells become more sensitive to cellular energy homeostasis. In addition, the ChIP-seq also revealed that TFE3 fusions could regulate cellular responses to hypoxia stress, which could improve cancer cell resistance to hypoxia and thus promote cancer growth. Therefore, these pathogenic mechanisms of TFE3 fusions may be potentially be exploited for the development of therapeutic targets.

\section{Conclusions}

In summary, we observed that overexpressed TFE3 fusions in Xp11.2 tRCC were capable of escaping from the control of the mTOR signaling pathway and showed evident nuclear retention. Inhibition of Gsk3 $\beta$ could increase cytoplasm retention of TFE3 fusions. In addition, TFE3 fusions could bind to target gene promoters as wild-type TFE3, promoting the expression of genes including lysosomal genes and hypoxia stress related genes, which could improve cell resistance against an extreme environment. Unveiling of these pathogenic mechanisms of TFE3 fusions may benefit the development of new cancer. 


\section{Additional files}

Additional file 1: Supplement S1. The primer sequences of mRNAs applied in this work. (TIF $176 \mathrm{~kb}$ )

Additional file 2: Supplement S2. (a) The fusion types of three renal carcinoma cell lines were detected by PCR. (b, c) In HK-2 and 786-O cells, expression of TFE3 (red) was examined by immunofluorescence microscopy after $24 \mathrm{~h}$ treatment with PP242. Nuclei were stained with DAPI (blue). Scale bar, 50 นm. (TIF 1403 kb)

Additional file 3: Supplement S3. Transcript-protein map of TFE3 aligned with NonO-TFE3 and PRCC-TFE3 fusions. Multiple sequence alignment highlight the phosphorylation site between wild-type TFE3, NonO TFE3 fusions and PRCC-TFE3 fusions. The breakpoint of the two fusion types were showed in blue words. The phosphorylation sites and the 143-3 proteins binding site of TFE3 were showed in red words. (PDF $75 \mathrm{~kb}$ )

Additional file 4: Supplement S4. The visualization data of ChIP-seq. Peaks were called using MACS version 2, with q-value 6set to 0.05. The horizontal axis of this chart is genomic location and the vertical axis represents bigwig. (TIF $1777 \mathrm{~kb}$ )

Additional file 5: Supplement S5. Predicted target genes of NonO-TFE3 in UOK109 cells from ChIP-seq. E-box sequence and distance from transcription start sites were analyzed using UCSC Genome Bioinformatics software. TSS, transcription start site. TTS, transcription terminal site. (XLSX $102 \mathrm{~kb}$ )

Additional file 6: Supplement S6. Predicted target genes of PRCC-TFE3 in UOK120 cells from ChIP-seq. E-box sequence and distance from transcription start sites were analyzed using UCSC Genome Bioinformatics software. TSS, transcription start site. TTS, transcription terminal site. (XLSX 29 kb)

\section{Abbreviations}

CCK-8: Cell counting kit-8; ChIP: Chromatin immunoprecipitation; GO: Gene Ontology; Gsk3ß: Glycogen synthase kinase 3 beta; KEGG: Kyoto Encyclopedia of Genes and Genomes; LAMP2: Lysosome-associated membrane protein 2; mTOR: mechanistic target of rapamycin; qRTPCR: Quantitative reverse transcription polymerase chain reaction; TFE3: Transcription factor binding to IGHM enhancer 3; tRCC: translocation renal cell carcinoma

\section{Acknowledgements}

Patient derived UOK cell lines were from Dr. Marston Linhan lab Urologic oncology Branch Tumor Cell Line Repository NCl. We give our sincere gratitude to Dr. W. Marston Linehan in National Cancer Institute for giving us the UOK109 and UOK120 cell lines.

\section{Funding}

This work was supported by National Natural Science Foundation of China (81572512), and Nanjing Sci-Tech Development Project (201803025).

\section{Availability of data and materials}

All data generated or analyzed during this study are included in this published article and its additional files. Additional datasets used and/or analyzed during the current study are available from the corresponding author on reasonable request.

\section{Authors' contributions}

$L D, Y X, G W$ and $H X$ conceived and designed the experiments. YX and WB performed the experiments and analyzed the data. GW, HX and LD contributed reagents/materials/analysis tools. YX, LD and XZ drafted the work and substantively revised it. ZW helped in data analysis and representation. All authors read and approved the final version of manuscript.

\section{Ethics approval and consent to participate}

The clinical tissue experiments were approved by Ethics Committee of the Affiliated Drum Tower Hospital of Medical School of Nanjing University.

\section{Consent for publication}

The author and participates are agree for publication.

\section{Competing interests}

The authors declare that they have no competing interests.

\section{Publisher's Note}

Springer Nature remains neutral with regard to jurisdictional claims in published maps and institutional affiliations.

\section{Author details}

${ }^{1}$ Immunology and Reproduction Biology Laboratory \& State Key Laboratory of Analytical Chemistry for Life Science, Medical School, Nanjing University, Nanjing 210093, Jiangsu, China. ${ }^{2}$ Jiangsu Key Laboratory of Molecular Medicine, Nanjing University, Nanjing 210093, Jiangsu, China. ${ }^{3}$ Department of Urology, Affiliated Drum Tower Hospital of Medical School of Nanjing University, Nanjing 210008, Jiangsu, China. ${ }^{4}$ Department of Health Technology and Informatics, Faculty of Health and Social Sciences, The Hong Kong Polytechnic University, Hung Hom, Kowloon, Hong Kong, China.

Received: 4 October 2018 Accepted: 11 February 2019

Published online: 08 March 2019

\section{References}

1. Lopez-Beltran A, Scarpelli M, Montironi R, Kirkali Z. 2004 WHO classification of the renal tumors of the adults. Eur Urol. 2006:49(5):798-805.

2. Moch H, Cubilla AL, Humphrey PA, Reuter VE, Ulbright TM. The 2016 WHO classification of Tumours of the urinary system and male genital organs-part a: renal, penile, and testicular Tumours. Eur Urol. 2016;70(1):93-105.

3. Clark J, Lu YJ, Sidhar SK, Parker C, Gill S, Smedley D, Hamoudi R, Linehan WM, Shipley J, Cooper CS. Fusion of splicing factor genes PSF and NonO (p54nrb) to the TFE3 gene in papillary renal cell carcinoma. Oncogene. 1997:15(18):2233-9.

4. Argani P, Antonescu CR, Couturier J, Fournet JC, Sciot R, Debiec-Rychter M, Hutchinson B, Reuter VE, Boccon-Gibod L, Timmons C, et al. PRCC-TFE3 renal carcinomas: morphologic, immunohistochemical, ultrastructural, and molecular analysis of an entity associated with the $t(X ; 1)(p 11.2 ; q 21)$. Am J Surg Pathol. 2002;26(12):1553-66.

5. Argani P, Lui MY, Couturier J, Bouvier R, Fournet JC, Ladanyi M. A novel CLTC-TFE3 gene fusion in pediatric renal adenocarcinoma with $t(X ; 17)(p 11.2$; q23). Oncogene. 2003;22(34):5374-8.

6. Ross H, Argani P. Xp11 translocation renal cell carcinoma. Pathology. 2010; 42(4):369-73.

7. Huang W, Goldfischer M, Babyeva S, Mao Y, Volyanskyy K, Dimitrova N, Fallon JT, Zhong M. Identification of a novel PARP14-TFE3 gene fusion from 10-year-old FFPE tissue by RNA-seq. Genes chromosomes \& cancer. 2015; 54(8):500-5

8. Argani P, Zhong M, Reuter VE, Fallon JT, Epstein JI, Netto GJ, Antonescu CR TFE3-fusion variant analysis defines specific Clinicopathologic associations among Xp11 translocation cancers. Am J Surg Pathol. 2016:40(6):723-37.

9. Sardiello M, Palmieri M, di Ronza A, Medina DL, Valenza M, Gennarino VA, Di Malta C, Donaudy F, Embrione V, Polishchuk RS, et al. A gene network regulating lysosomal biogenesis and function. Science. 2009;325(5939):473-7.

10. Martina JA, Diab HI, Lishu L, Jeong AL, Patange S, Raben N, Puertollano R. The nutrient-responsive transcription factor TFE3 promotes autophagy, lysosomal biogenesis, and clearance of cellular debris. Sci Signal. 2014; 7(309):ra9.

11. Settembre C, Di Malta C, Polito VA, Garcia Arencibia M, Vetrini F, Erdin S, Erdin SU, Huynh T, Medina D, Colella P, et al. TFEB links autophagy to lysosomal biogenesis. Science. 2011;332(6036):1429-33.

12. Roczniak-Ferguson A, Petit CS, Froehlich F, Oian S, Ky J, Angarola B, Walther TC, Ferguson SM. The transcription factor TFEB links mTORC1 signaling to transcriptional control of lysosome homeostasis. Sci Signal. 2012;5(228):ra42.

13. Settembre C, Zoncu R, Medina DL, Vetrini F, Erdin S, Erdin S, Huynh T, Ferron M, Karsenty G, Vellard MC, et al. A lysosome-to-nucleus signalling mechanism senses and regulates the lysosome via mTOR and TFEB. EMBO J. 2012;31(5):1095-108.

14. Kauffman EC, Ricketts CJ, Rais-Bahrami S, Yang Y, Merino MJ, Bottaro DP Srinivasan R, Linehan WM. Molecular genetics and cellular features of TFE3 and TFEB fusion kidney cancers. Nature reviews Urology. 2014;11(8):465-75.

15. Argani P, Antonescu CR, Illei PB, Lui MY, Timmons CF, Newbury R, Reuter VE, Garvin AJ, Perez-Atayde AR, Fletcher JA, et al. Primary renal neoplasms with the ASPL-TFE3 gene fusion of alveolar soft part sarcoma. a distinctive tumor 
entity previously included among renal cell carcinomas of children and adolescents Am J Pathol. 2001;159(1):179-92.

16. Hua X, Liu X, Ansari DO, Lodish HF. Synergistic cooperation of TFE3 and smad proteins in TGF-beta-induced transcription of the plasminogen activator inhibitor-1 gene. Genes Dev. 1998;12(19):3084-95.

17. Tian G, Erman B, Ishii H, Gangopadhyay SS, Sen R. Transcriptional activation by ETS and leucine zipper-containing basic helix-loop-helix proteins. Mol Cell Biol. 1999;19(4):2946-57.

18. Huan C, Kelly ML, Steele R, Shapira I, Gottesman SR, Roman CA. Transcription factors TFE3 and TFEB are critical for CD40 ligand expression and thymusdependent humoral immunity. Nat Immunol. 2006;7(10):1082-91.

19. Argani P, Hicks J, De Marzo AM, Albadine R, Illei PB, Ladanyi M, Reuter VE, Netto GJ. Xp11 translocation renal cell carcinoma (RCC): extended immunohistochemical profile emphasizing novel RCC markers. Am J Surg Pathol. 2010;34(9):1295-303.

20. Anglard P, Trahan E, Liu S, Latif F, Merino MJ, Lerman MI, Zbar B, Linehan WM. Molecular and cellular characterization of human renal cell carcinoma cell lines. Cancer Res. 1992;52(2):348-56.

21. Chen $X, X u H$, Yuan P, Fang F, Huss M, Vega VB, Wong E, Orlov YL, Zhang WW, Jiang $J M$, et al. Integration of external signaling pathways with the core transcriptional network in embryonic stem cells. Cell. 2008;133(6):1106-17.

22. Zhang Y, Liu T, Meyer CA, Eeckhoute J, Johnson DS, Bernstein BE, Nussbaum C, Myers RM, Brown M, Li W, et al. Model-based analysis of ChIPSeq (MACS). Genome Biol. 2008;9(9):R137.

23. Ploper D, Taelman VF, Robert L, Perez BS, Titz B, Chen HW, Graeber TG, von Euw E, Ribas A, De Robertis EM. MITF drives endolysosomal biogenesis and potentiates Wnt signaling in melanoma cells. Proc Natl Acad Sci U S A. 2015;112(5):E420-9.

24. Puertollano R, Ferguson SM, Brugarolas J, Ballabio A. The complex relationship between TFEB transcription factor phosphorylation and subcellular localization. EMBO J 2018; 37(11). pii: e98804.

25. Tsukumo Y, Alain T, Fonseca BD, Nadon R, Sonenberg N. Translation control during prolonged mTORC1 inhibition mediated by 4E-BP3. Nat Commun. 2016;7:11776.

26. Betschinger J, Nichols J, Dietmann S, Corrin PD, Paddison PJ, Smith A. Exit from pluripotency is gated by intracellular redistribution of the bHLH transcription factor Tfe3. Cell. 2013:153(2):335-47.

27. Raben N, Puertollano R. TFEB and TFE3: linking lysosomes to cellular adaptation to stress. Annu Rev Cell Dev Biol. 2016:32:255-78.

28. Pastore N, Brady OA, Diab HI, Martina JA, Sun L, Huynh T, Lim JA, Zare H, Raben $\mathrm{N}$, Ballabio $\mathrm{A}$, et al. TFEB and TFE3 cooperate in the regulation of the innate immune response in activated macrophages. Autophagy. 2016;12(8):1240-58

29. Weterman MA, van Groningen JJ, den Hartog A, Geurts van Kessel A. Transformation capacities of the papillary renal cell carcinoma-associated PRCCTFE3 and TFE3PRCC fusion genes. Oncogene. 2001;20(12):1414-24.

30. Weterman MAJ, von Groningen JJM, Jansen A, van Kessel AG. Nuclear localization and transactivating capacities of the papillary renal cell carcinoma-associated TFE3 and PRCC (fusion) proteins. Oncogene. 2000; 19(1):69-74

31. Li Y, Xu M, Ding X, Yan C, Song Z, Chen L, Huang X, Wang X, Jian Y, Tang G, et al. Protein kinase $C$ controls lysosome biogenesis independently of mTORC1. Nat Cell Biol. 2016;18(10):1065-77.

32. Ploper D, De Robertis EM. The MITF family of transcription factors: role in endolysosomal biogenesis, Wnt signaling, and oncogenesis. Pharmacol Res. 2015;99:36-43

33. Ferron M, Settembre C, Shimazu J, Lacombe J, Kato S, Rawlings DJ, Ballabio A, Karsenty G. A RANKL-PKCbeta-TFEB signaling cascade is necessary for lysosomal biogenesis in osteoclasts. Genes Dev. 2013;27(8):955-69.

34. Lim CY, Zoncu R. The lysosome as a command-and-control center for cellular metabolism. J Cell Biol. 2016;214(6):653-64.

Ready to submit your research? Choose BMC and benefit from:

- fast, convenient online submission

- thorough peer review by experienced researchers in your field

- rapid publication on acceptance

- support for research data, including large and complex data types

- gold Open Access which fosters wider collaboration and increased citations

- maximum visibility for your research: over $100 \mathrm{M}$ website views per year

At BMC, research is always in progress.

Learn more biomedcentral.com/submissions 